\title{
Letter to the Editor: The bioenergetics of hepatic mitochondria isolated from avocado oil-treated rats: typical experimental errors in the study of the bioenergetics of isolated mitochondria
}

\author{
Dairo A. Rendon ${ }^{1}$
}

Received: 25 August 2015 / Accepted: 14 October 2015 /Published online: 29 October 2015

(C) Springer Science+Business Media New York 2015

In the article by Ortiz-Avila et al. (2015) entitled "Protective effects of dietary avocado oil on impaired electron transport chain function and exacerbated oxidative stress in liver mitochondria from diabetic rats", published in the journal $J$ Bioenerg Biomembr, June 10, 2015 [Epub ahead of print], (J Bioenerg Biomembr, August 2015, Volume 47, Issue 4, pp 337-353, DOI 10.1007/s10863-015-9614-z), the authors consider that the regular consumption of avocado oil by otherwise normal persons with a predisposition toward diabetes is beneficial for the protection of these persons against a possible hepatic dysfunction on the mitochondrial level. (See the first paragraph, lines 12-16, of the last page of the text: "Finally, other possibility is that regular consumption of avocado oil in the diet may delay the development of liver dysfunction in people with predisposition to suffer metabolic syndrome before the clinical and biochemical manifestations of this disease begin"). Nevertheless, based on the experimental data published in that article (Fig. 8a and 8b), it can clearly be seen that this could be counterproductive for normal hepatic functioning. This opinion is based on the following: the functional integrity of the mitochondria is crucial for the bioenergetics of the cells of the liver and other organs. The easiest and most effective way to experimentally determine the functional integrity of isolated mitochondria is by measuring the respiratory control, RC (Rickwood et al. 1987).

An author's reply to this comment is available at doi:10.1007/s10863015-9629-5.

Dairo A. Rendon

darendon@unal.edu.co

1 Facultad de Ciencias, Escuela de Física, Laboratorio de Biofísica, Universidad Nacional de Colombia-Sede Medellin, AA 3840, Calle 59 A No 63-020, Autopista Norte, Medellín, Colombia
Ortiz-Avila et al. (2015) did not report the RC of the isolated mitochondria from rat livers, but nevertheless an average of this parameter can be calculated on the basis of the data for state 2 and state 3 reported in this investigation (Figure $8 \mathrm{~b}$ ), since $\mathrm{RC}=$ state $2 /$ state 4. In this way, according to Figure $8 \mathrm{~b}$, the mitochondria isolated from the livers of normal rats that were subjected to a daily diet (for 90 days) rich in avocado oil exhibited an average $\mathrm{RC}=1.1$ (versus an average $\mathrm{RC}=1.7$ for the hepatic mitochondria isolated from normal rats). That is to say, the mitochondria isolated from the livers of normal rats subjected to the aforementioned diet are totally uncoupled, indicating that these mitochondria are not capable in practice of synthesizing ATP from ADP (this is confirmed by the curve "Control + AO" shown in Figure 8a). There are two possible causes of this mitochondrial uncoupling. A) It is possible that in the natural composition of the avocado or in the components used in the preparation of this avocado oil (Ahuacatlan, DIRICOM, S. A. de C.V., México) there exists some kind of uncoupler of the hepatic mitochondria, which would be exercising its mitochondrial uncoupling action in vivo; in this case, the continued consumption of avocados and/or the avocado oil used in this study would be counterproductive for the normal functioning of the liver of normal persons, because it would negatively impact the mitochondrial bioenergetics of this organ. B) Another possible cause is that this mitochondrial uncoupling observed in the data of Figures $8 \mathrm{a}$ and $8 \mathrm{~b}$ was the fruit of some experimental artefact, and according to the curves of the record of oxygen consumption by the isolated hepatic mitochondria, reported in Figure $8 \mathrm{a}$, there is reason not to discard this possibility: 1) The isolated mitochondria remained in state 2 for five minutes (see the section "Determination of the activities of the ETC complexes and mitochondrial respiration"). This incubation time is a lot when one is experimenting with isolated mitochondria, and thus possibly the mitochondria were allowed to begin to undergo additional (negative) structural modifications. It 
could be that for this reason that for the control mitochondria and for the mitochondria of rats fed a supplement of avocado oil, state 2 becomes inhibited with the passing of the recorded time (see the curves "Control" and Control + AO" in Figure 8a). The slope of these curves fell during the last minute of state 2 by approximately $55 \%$ with respect to the first minute. All of the above is indicative of possible experimental artefacts that led to an average $\mathrm{RC}=1.1$ for the mitochondria isolated from the livers of normal rats that were subjected to the aforementioned diet rich in avocado oil. Another possible explanation for the above-mentioned fall in the slope of state 2 in the curves "Control" and "Control + AO" of Figure $8 \mathrm{a}$ is that air (oxygen) was entering through the cell. In this case, the rate of possible entry of oxygen is greater, with respect to the first minute, in the last minute of state 2 , because over the course of the recorded time, the gradient between the concentrations of oxygen outside of and inside of the cell increases (since the mitochondria use up the oxygen inside of the cell). In order to check this possibility, it is necessary to record the consumption of oxygen for more or less two minutes when there is only incubation buffer in the cell (the so-called state 1, see (Nicholls and Ferguson 2001). This state 1 was not shown in Figure 8a); in this case, the recorded slope should be zero. In order to avoid all of these possible experimental artefacts, the best thing is to record state 1 for more or less two minutes and confirm a recorded slope of zero; then induce state 2 and record it for more or less a minute and a half (the stationary state for state 2 is established very quickly once the oxidative substrate is added, see Figure 8a). And without fail do not take into account the record in which changes in the slope of state 2 are seen, because that could be an indication that the isolated mitochondria are undergoing additional (negative) structural changes, product of experimental manipulation and/or of contamination of the incubation medium, etc.

Finally, RC is an indicator of the degree of energetic coupling of the isolated mitochondria, and its numeric value obtained for a suspension of mitochondria isolated from normal animals is an indicator of how well or how badly the mitochondria were isolated and/or the experiments were set up for the determination of the consumption of oxygen. Typical values for isolated-mitochondria $\mathrm{RC}$ from livers of normal rats, using glutamate plus malate as an oxidative substrate and an incubation buffer with low ionic strength, are on the order of RC $=10-16$, see reference (Rickwood et al. 1987), and for the aforementioned experimental conditions, except that in an incubation buffer with high ionic strength, the RC is on the order of $\mathrm{RC}=9$, see reference (Rendon and AlvarezBustamante 2011). Ortiz-Avila et al. (2015) obtained an average value of $\mathrm{RC}=1.7$ for isolated-mitochondria $\mathrm{RC}$ of the livers of normal rats, using glutamate plus malate as oxidative substrate and an incubation buffer with high ionic strength. This average value is very low with respect to the values reported in other papers (Rickwood et al. 1987; Rendon and
Alvarez-Bustamante 2011), this being an additional indicator that during the isolation and/or experimental determination of the oxygen consumption by the mitochondria isolated from the livers of normal rats carried out by Ortiz-Avila et al. (2015) experimental artefacts appeared that led to obtaining isolated mitochondria with a low degree of energetic coupling. It is possible that all of these above-mentioned experimental artefacts more severely impacted the mitochondria isolated from the livers of normal rats fed on avocado oil than those obtained from normal rats, and for that reason an average $\mathrm{RC}=1.1$ was observed for avocado oil-treated rats versus an average $\mathrm{RC}=1.7$ for normal rats.

The probability of the existence of a possible mitochondrial uncoupler in the natural composition of avocados and/or in the composition of the substances used for obtaining the avocado oil (Ahuacatlan, DIRICOM, S.A. de C.V., México) declines when it is observed that the mitochondria isolated from the livers of diabetic rats that received a repetitive diet rich in the avocado oil are not uncoupling (average $\mathrm{RC}=2.6$ ). This indicates that the most appropriate thing is to seek the possible causes of an average $\mathrm{RC}=1.1$ for the mitochondria isolated from the livers of normal rats that were subjected to a diet rich in avocado oil in some experimental artifact that appeared during the isolation and/or experimental determination of the oxygen consumption by the hepatic mitochondria isolated from rats.

Diabetes is a disease that more and more affects a greater percentage of the world population, and every research study in basic science aimed toward seeking possible therapeutic treatments for this disease is very valuable. But these studies should be characterized by great scientific rigor, in order to successfully produce a possible partial or total solution for this problem in the future. Because of this, it is pertinent to clarify if the average $\mathrm{RC}=1.1$ obtained by Ortiz-Avila et al. (2015) for mitochondria isolated from the livers of normal rats that were subjected to a daily diet (for 90 days) rich in avocado oil is because the hepatic mitochondria are the target in vivo of some mitochondrial uncoupler present in avocados and/or in avocado oil (Ahuacatlan, DIRICOM, S.A. de C.V., México), or rather this was due to experimental artefacts that appeared during the in vitro manipulation of the isolated mitochondria.

\section{References}

Ortiz-Avila O, Gallegos-Corona MA, Sánchez-Briones LA, CalderónCortés E, Montoya-Pérez R, Rodriguez-Orozco AR, CamposGarcía J, Saavedra-Molina A, Mejía-Zepeda R, Cortés-Rojo C (2015). Protective effects of dietary avocado oil on impaired electron transport chain function and exacerbated oxidative stress in liver mitochondria from diabetic rats. J Bioenerg Biomembr 47(4):337353. doi:10.1007/s10863-015-9614-z

Rickwood D, Wilson MT, Darley-Usmar VM. Isolation and characteristics of intact mitochondria. In: Darley-Usmar VM, Rickwood D, 
Wilson MT, Eds. Mitochondria a practical approach. 1st ed. Oxford: IRL Press Limited 1987; pp. 1-33.

Nicholls DG, Ferguson SJ (2001) The chemiosmotic proton circuit. In: Nicholls DG, Ferguson SJ (eds) Bioenergetics 3, 4th ed. Academic Press, San Diego, pp. 57-87
Rendon DA, Alvarez-Bustamante JA (2011) Early hyperglycemia following alloxan administration in vivo is not associated with altered hepatic mitochondrial function: acceptable model for type 1 diabetes? Can J Physiol Pharmacol 89:477-484 